\title{
An epidemiological description of spinal cord injuries in The Netherlands in 1994
}

\author{
FWA van Asbeck ${ }^{*}, 1$, MWM Post ${ }^{1}$ and RF Pangalila ${ }^{1}$ \\ ${ }^{1}$ Spinal Cord Injury Unit, "De Hoogstraat" Rehabilitation Centre, Utrecht, The Netherlands
}

\begin{abstract}
Study design: Retrospective descriptive study.
Objectives: To ascertain the incidence of spinal cord injury in The Netherlands.

Methods: From all patients, discharged from a Dutch general hospital in 1994 with the ICD9 diagnosis code 806 (fracture of the spine with injury of the spinal cord) and 952 (injury to the spinal cord without apparent spinal fracture), a copy of the anonymized medical correspondence was requested. The received correspondence was analyzed for the diagnosis of traumatic spinal cord injury with motor, sensory, bladder and bowel symptoms lasting longer than 2 weeks.
\end{abstract}

Results: 479 cases with the ICD-9 codes 806 or 952 were identified. On 329 cases we received information. According to our criteria 126 cases had a spinal cord injury with persisting symptoms. Of these 126 cases 18 died during hospital stay. Sex ( $77 \%$ male), level (57\% tetra), completeness ( $48.7 \%$ complete), age distribution, cause of injury and incidence of stabilizing operation $(44.2 \%)$ were assessed. Mean hospital stay was 31 days and of the subjects who survived the initial hospital phase, $70 \%$ were referred to a rehabilitation centre or a rehabilitation ward.

Conclusion: The incidence of spinal cord injury surviving the acute phase in The Netherlands in 1994 was $10.4 /$ million/annum.

Sponsorship: De Hoogstraat Scientific Foundation.

Spinal Cord (2000) 38, 420-424

Keywords: spinal cord injury; traumatic; epidemiology; incidence; The Netherlands

\section{Introduction}

As yet, little is known about the incidence of spinal cord injuries in The Netherlands. Schönherr et al published a study based on patients with a spinal cord lesion in a rehabilitation centre; they tentatively estimated the incidence of spinal cord lesions traumatic and non-traumatic, to be 16 per million inhabitants per year, but they assumed that nearly all traumatic spinal cord injured patients would be transferred to a rehabilitation centre and noted that a reliable registration of the group with non-traumatic lesions is unavailable.

There are no other known studies of incidence of spinal cord injuries in The Netherlands. ${ }^{1,2}$

In The Netherlands, spinal cord injured patients are first admitted to a general hospital; from there, they are transferred to a rehabilitation institution.

A national hospital registration of disease does exist in The Netherlands, but there was a big discrepancy in the numbers of spinal cord injured patients found in this registration and the numbers found in the

*Correspondence: FWA van Asbeck rehabilitation centres. In 1987 the number of spinal cord injured patients in the national hospital registration exceeded 500, whereas there were no more than 120 patients found in the rehabilitation centres.

The international literature, the incidence figures are widely variable, from 9 to 53 per million inhabitants per year $(3-11)$.

In this study we tried to give an epidemiological description of the group of spinal cord injured patients in The Netherlands. Specifically, we were interested in the incidence of spinal cord injuries, the percentage of spinal cord injured patients that were transferred to a rehabilitation institution and an explanation for the discrepancy in the number of spinal cord injured patients found in the national registration and the number found in the rehabilitation centres.

\section{Materials and methods}

In The Netherlands there exists a national registration for disease, based on the International Classification of Diseases (ICD-9); this registration is managed by an independent organization (SIG Zorginformatie, Utrecht, The Netherlands). All patients who are 
discharged from a Dutch hospital are coded in this registration system. Traumatic spinal cord injuries are to be found in two respective codes: 806 (fracture of the spine with injury of the spinal cord) and 952 (injury to the spinal cord without apparent spinal fracture). In this retrospective, descriptive study for practical reasons we decided to analyze all cases registered in 1994.

We selected all cases that were registered with either codes 806 or 952 in the main or concurrent diagnoses in 1994. For each of these cases, the registering hospital was approached through SIG with a request for the anonymized medical correspondence regarding all cases, explaining that the information would be used in an epidemiological study.

If correspondence was obtained, we analyzed it for diagnosis. Spinal cord injury was diagnosed if there was an acquired traumatic transverse lesion of the spinal cord and/or cauda equina, resulting in loss of motor, sensory, bladder and bowel function below the level of the lesion and loss of motor function was still present after a period of 2 weeks. If there were only transient neurological symptoms or pain, we diagnosed a contusion of the spinal cord. If a spinal cord injury was diagnosed, the correspondence was further analyzed for gender, type of lesion, age, cause of injury, stabilizing operations and discharge destination.

\section{Results}

In 1994,479 cases with either codes 806 or 952 were registered in the national hospital registration system. We received the actual correspondence concerning 256 cases $(53.5 \%)$. In 73 cases $(15.2 \%)$ no actual correspondence was obtained but the referring specialist indicated that there was no spinal cord injury on reevaluation (60 cases) or just indicated that there was an actual diagnosis of spinal cord injury (13 cases). In 35 cases $(7.3 \%)$ cooperation was refused for reasons of time-intensity or because warrant for the anonymity of the requested information was deemed insufficient. In 115 cases $(24.0 \%)$ no reply was received at all.

Of the 256 cases for whom correspondence was obtained, 113 proved to be actual new spinal cord injuries with persistent neurological symptoms. In another 113 cases there was no spinal cord injury according to our definition; among these, 66 were diagnosed as contusions of the spinal cord with no or quickly passing neurological symptoms (often paresthesia or pain) and 22 cases had an injury of the spine without neurological symptoms. Among others, we also found plexus lesions and intervertebral disc herniations in this group. Of the remaining 30 cases, 25 concerned pre-existing spinal cord injuries or a second registration of an already known case. In five cases it was impossible to adequately analyze the correspondence.

Thus, we obtained information about 329 cases, of which 126 proved to have an actual new spinal cord injury.
Assuming that the ratios in the known group and the total group are the same, the total number of spinal cord injuries for the year 1994 in The Netherlands would be approximated by (126/ $329) \times 479=183.4$. In the Dutch population of 15.1 million this corresponds with an incidence of 12.1/ million/annum for all spinal cord injuries. Since 18 patients died during their hospital stay the number of spinal cord injury surviving the acute phase in The Netherlands in 1994 was $(108 / 329) \times 479=157.2$. This corresponds with an incidence of $10.4 / \mathrm{million} /$ annum for spinal cord injuries surviving the acute phase.

In the group of 113 spinal cord injuries for whom analyzable correspondence was obtained, further analysis was carried out. Of these subjects, 87 $(77.0 \%)$ were male and $26(23.0 \%)$ were female, giving a male : female ratio of $3.3: 1$.

The distribution of cases with complete and incomplete lesions and tetraplegia and paraplegia is shown in Table 1 . In the tetraplegic group 23 subjects were found with pre-existing spondylarthropathy; most of these were elderly patients.

The distribution of age is shown in Table 2. The cause of injury is shown in Table 3. 'Fall' includes both falls from height and same-level falls.

The destination of patients leaving the hospital phase is shown in Table 4. Eighteen subjects died in the hospital phase. Pulmonary problems were assessed as cause of death in eight of these patients. Sixty-six subjects $(58.4 \%)$ were referred to a rehabilitation clinic or a rehabilitation ward in a general hospital. One of these subjects immediately started in an outpatient programme. Of the subjects who survived the hospital phase, $69.5 \%$ were referred to a rehabilitation clinic or

Table 1 SCI cases in 1994 for whom correspondence was obtained. Distribution of completeness and level

\begin{tabular}{|c|c|c|c|c|c|c|}
\hline & \multicolumn{2}{|c|}{ Tetra } & \multicolumn{2}{|c|}{ Para } & \multicolumn{2}{|c|}{ Total } \\
\hline Complete & 26 & & 29 & & 55 & $(48.7 \%)$ \\
\hline Incomplete & 39 & & 19 & & 58 & $(51.3 \%)$ \\
\hline Total & 65 & $(57.5 \%)$ & 48 & $(42.5 \%)$ & 113 & \\
\hline
\end{tabular}

Table 2 SCI cases in 1994 of whom correspondence was obtained. Age distribution

\begin{tabular}{lrr}
\hline Age (years) & & \\
\hline $0-10$ & 2 & $(1.8 \%)$ \\
$11-20$ & 13 & $(11.5 \%)$ \\
$21-30$ & 28 & $(24.8 \%)$ \\
$31-40$ & 16 & $(14.1 \%)$ \\
$41-50$ & 12 & $(10.6 \%)$ \\
$51-60$ & 8 & $(7.1 \%)$ \\
$61-70$ & 11 & $(9.7 \%)$ \\
$71-80$ & 18 & $(15.9 \%)$ \\
$>80$ & 5 & $(4.4 \%)$ \\
Total & 113 & \\
\hline
\end{tabular}


Table 3 SCI cases in 1994 of whom correspondence was obtained. Distribution of cause of injury

\begin{tabular}{|c|c|c|c|c|c|}
\hline \multicolumn{6}{|l|}{ Cause } \\
\hline Traffic & 35 & $(31.0 \%)$ & $\begin{array}{l}\text { Bicycle } \\
\text { Moped } \\
\text { Motorcycle } \\
\text { Car }\end{array}$ & $\begin{array}{r}6 \\
3 \\
8 \\
18\end{array}$ & $\begin{array}{l}(5.3 \%) \\
(2.7 \%) \\
(7.1 \%) \\
(15.9 \%)\end{array}$ \\
\hline Occupational & 5 & $(4.4 \%)$ & & & \\
\hline Sports & 10 & $(8.9 \%)$ & $\begin{array}{l}\text { Diving } \\
\text { Other }\end{array}$ & $\begin{array}{l}7 \\
3\end{array}$ & $\begin{array}{l}(6.2 \%) \\
(2.7 \%)\end{array}$ \\
\hline $\begin{array}{l}\text { Fall } \\
\text { Other } \\
\text { Unknown } \\
\text { Total }\end{array}$ & $\begin{array}{r}55 \\
4 \\
4 \\
113\end{array}$ & $\begin{array}{c}(48.7 \%) \\
(3.5 \%) \\
(3.5 \%) \\
(100 \%)\end{array}$ & & & \\
\hline
\end{tabular}

Table 4 SCI cases in 1994 of whom correspondence was obtained. Distribution of destination

\begin{tabular}{lrr}
\hline Destination & & \\
\hline Home & 8 & $7.1 \%$ \\
Other hospital & 9 & $8.0 \%$ \\
Nursing home & 10 & $8.8 \%$ \\
Rehabilitation clinic & 60 & $53.1 \%$ \\
Rehabilitation clinic outpatient & 1 & $0.9 \%$ \\
Rehabilitation ward hospital & 5 & $4.4 \%$ \\
Dead & 18 & $15.9 \%$ \\
Unknown & 2 & $1.8 \%$ \\
Total & 113 & \\
\hline
\end{tabular}

a rehabilitation ward. Mean hospital stay was 30.9 days (range: 6-118 days). The nine cases who were referred to another hospital were checked for double registration but nothing was found. They probably went to hospitals that did not correspond. In Table 5 the distribution of the types of lesion over the destinations is shown.

Fifty subjects $(44.2 \%)$ received a stabilizing operation following their spinal cord injury. Whether or not a patient received an operation was not significantly correlated to completeness of the lesion or the level of spinal cord injury.

\section{Discussion}

Knowledge of the epidemiology of spinal cord injury in a given country is important, if only for the planning of the resources, needed for adequate treatment and rehabilitation. $^{12}$

The incidence of spinal cord injury of $10.4 /$ million inhabitants/annum, found in this study was concordant with figures found in other countries in Europe, which vary between 9 and 16/million/annum. ${ }^{4,5,7,8}$ Schönherr et $a l^{1}$ estimated the incidence in their region of the northern Netherlands to be $16 /$ million/ annum in the rehabilitation centres, based on the number of cases seen in their centre. Of these, about $50 \%$ were traumatic. This gives an incidence of $8 /$ million/annum for the traumatic lesions.
In our study we found that only $69.5 \%$ of the surviving patients went to a rehabilitation institution; possibly the cases with unknown discharge destination from the hospital, and those cases referred to another hospital eventually were transferred to a rehabilitation centre, thus giving a maximum of $81 \%$ of patients with spinal cord injury who were transferred to a rehabilitation institution. In this perspective, the incidence figures as estimated by Schönherr et $a l^{1}$ and the $10.4 / \mathrm{million} /$ annum found in our study are concordant.

The incidence figures found worldwide vary widely. In part, this is due to local demographic and socioeconomic factors. ${ }^{10}$ Other important factors seem to be the methodological differences in estimating the incidence and the type of registration used. For instance, studies sometimes concentrate on spinal cord injuries, ${ }^{3-6,8-11}$ but other studies include all spinal cord lesions, irrespective of etiology. 1,7

Also, in some studies only patients in rehabilitation centres are included, ${ }^{1,4,5,7}$ whereas in other studies the sample exists of all patients in a given period of time. 6,8-11 Considering our findings about the percentage of patients that enter a rehabilitation centre, this leads to big differences in the incidence figures found.

The type of registration seems to be of a great influence. In our study, we started with those cases, registered in The Netherlands in 1994 with ICD-9codes 806 (spinal fracture with damage to the spinal cord) and 952 (spinal cord damage without apparent fracture of the spine). When we compared the registration data with the diagnoses we found either in the medical correspondence concerning these cases or obtained by notification of the diagnosis by the referring hospital, that no more than 126 of 329 cases $(38.3 \%)$ had a new spinal cord injury with persisting neurological symptoms.

In many cases, the actual diagnosis proved to be a contusion of the spinal cord with transient neurological symptoms, but also other diagnoses (among others fracture of the spine without neurological symptoms and lesions of brachial or solar plexus) were found. Thus, an incidence figure based on the codes 806 and 
Table 5 SCI cases in 1994 of whom correspondence was obtained with known destination. Type of SCI vs destination

\begin{tabular}{lcccc}
\hline & Rehabilitation & Other & Dead & Total \\
\hline Tetra/complete & $10(40 \%)$ & $4(16 \%)$ & $11(44 \%)$ & 25 \\
Tetra/incomplete & $23(59 \%)$ & $13(33 \%)$ & $3(8 \%)$ & 39 \\
Para/complete & $20(69 \%)$ & $5(17 \%)$ & $4(14 \%)$ & 29 \\
Para/incomplete & $13(72 \%)$ & $5(18 \%)$ & $0(0 \%)$ & 18 \\
\hline
\end{tabular}

952 was, by our definition, hugely overestimated. Dixon et $a l^{6}$ based an epidemiological study in New Zealand on the ICD codes 806 and 952. They found a high incidence $(49.1 / \mathrm{mln} /$ annum $)$, but also a strikingly high percentage of incomplete lesions (92\%). Possibly this was due to a large number of spinal cord contusions in their sample. The Japanese epidemiological studies by Shingu et $a l^{10,11}$ registered all cases coded with ICD 806 and 952, but they excluded the Frankel $\mathrm{E}$ cases in their estimation of incidence.

Based on our findings, we conclude that the assumption that, in The Netherlands practically every spinal cord injured patient is transferred to a rehabilitation institution, is false. Of the 95 patients surviving the hospital phase in our sample $69.5 \%$ were referred to a rehabilitation facility, with a maximum of $81 \%$, if patients in our sample who went to another hospital or to unknown destinations are included.

In our opinion, patients with tetraplegia and paraplegia should be concentrated in a small number of institutions for their rehabilitation, since their absolute number is small and concentration in regionalized centres would give these institutions the possibility to gain and maintain experience in the management of spinal cord injured patients. In The Netherlands in 1994, these patients were rehabilitated in eight rehabilitation institutions with a specialized ward, with some going to a number of smaller institutions.

The male-female ratio of $3.3: 1$ found in our study is consistent with ratios found in other studies ${ }^{4-11}$ and is exactly the same as the ratio found by Schönherr et $a l^{1}$ in their traumatic group.

The age distribution in our study shows a peak in the 21-30 years of age group $(24.8 \%)$, but also a relatively large number of patients in the elderly group ( $71-80$ years: $15.9 \%)$. In most studies a relatively high risk in those having a spinal cord injury is found in the age group of $15-30$ years. $^{4-9}$ The smaller peak in the elderly group in our study could be ascribed to a group of 23 patients $(20.4 \%)$ with pre-existing spondylarthropathy, for the most part elderly patients who had a relatively minor trauma resulting in an incomplete tetraplegia.

In our study, we found $57.5 \%$ cervical lesions. Again, this is consistent with percentages found in other European studies. ${ }^{1,4,5,7,8}$ The percentage of complete lesions $(48.7 \%)$ is also consistent with the findings in these studies.

The cause of injury was a fall in $48.7 \%$ and a traffic accident in $31 \%$. In international literature, the distribution of the cause of injury is found to be strongly dependant on local factors, for instance concerning the level of violence or intensity of traffic. $^{4-11}$ The high percentage of injuries due to a fall in our study again is influenced by the group of patients with pre-existing spondylarthropathy, who often had a same-level fall.

In the management of spinal cord injury in The Netherlands, there is some debate about the benefit of a stabilising operation. Possible advantages are a prevention of progressing neurological symptoms in incomplete lesions and the possibility of quickly mobilizing the post operative patients.

The disadvantage, especially for paraplegic patients, is the extra loss of mobility of the spine due to a spondylodesis, possibly augmenting the disabilities in their transfer and the activities of daily living. In our study, $44 \%$ of the patients received a stabilizing operation. There was no significant relationship between the result of an operation and either completeness or level of the lesion.

\section{Conclusions}

In this study, we found an incidence of $12.1 /$ million inhabitants/annum for all spinal cord injuries in The Netherlands and 10.4 /million inhabitants/annum if only the spinal cord injuries surviving the acute phase were counted.

The differences in incidence figures elsewhere in the world seem partly due to methodological differences in the studies. We found that using the ICD-9 codes 806 and 952 led to a large overestimation of the incidence of spinal cord injury with persisting neurological damage. Of the spinal cord injured patients surviving the acute phase, $69.5 \%$ were eventually transferred to a rehabilitation institution.

In our opinion it is important to concentrate spinal cord injured patients to a small number of specialized institutions for their rehabilitation, because the small absolute number of patients (estimated to be about 154 spinal cord injuries discharged per year alive from the Dutch hospitals), sufficient experience will be gained in the treatment and maintenance of spinal cord injured patients.

\section{References}

1 Schönherr MC, Groothoff JW, Mulder GA, Eisma WH Rehabilitation of patients with spinal cord injuries in The Netherlands; an epidemiological study. Spinal Cord 1996; 34: $679-683$. 
2 van Asbeck FWA. Functioneren met een paraplegie. Thesis, University of Groningen; Groningen, The Netherlands, 1987.

3 Blumer CE, Quine S. Prevalence of spinal cord injury: an international comparison. Neuroepidemiology 1995; 14: $258-$ 268.

4 Biering-Sørensen SF, Pedersen V, Clause S. Epidemiology of spinal cord lesions in Denmark. Paraplegia 1990; 28: 105-118.

5 Dincer $\mathrm{F}$ et al. Traumatic spinal cord injuries in Turkey. Paraplegia 1992; 30: $641-646$.

6 Dixon GS, Danesh JN, Caradoc-Davies TH. Epidemiology of spinal cord injury in New Zealand. Neuroepidemiology 1993; 12: $88-95$.

7 Garcia-Reneses J, Herruzo-Cabrera R, Martinez-Moreno M. Epidemiological study of spinal cord injury in Spain 1984-1985. Paraplegia 1991; 28: 180-190.
8 Minaire $\mathrm{P}$ et al. Epidemiology of spinal cord injury in the RhoneAlpes region, France, 1970-75. Paraplegia 1978/1979; 16: $76-$ 87

9 Price C, Makintubee S, Herndon W, Istre GR. Epidemiology of traumatic spinal cord injury and acute hospitalization and rehabilitation charges for spinal cord injuries in Oklahoma, 1988 - 1990. American Journal of Epidemiology 1994; 139: 37 - 47.

10 Shingu H, Ikata T, Katoh S, Akatsu T. Spinal cord injuries in Japan; a nationwide epidemiological survey in 1990. Paraplegia 1994; 32: 3-8.

11 Shingu $\mathrm{H}$ et al. A nationwide epidemiological survey of spinal cord injuries in Japan from January 1990 to December 1992 Paraplegia 1995; 33: $183-188$

12 Lasfargues JE et al. A model for estimating spinal cord injury prevalence in the United States. Paraplegia 1995; 33: 62-68. 